\title{
Intensification prépositionnelle et préfixationnelle
}

\author{
Silvia Adler \\ Université de Haïfa \\ sadler@univ.haifa.ac.il \\ Maria Asnes \\ Université de Bar Ilan \\ maria.asnes@gmail.com
}

\section{Introduction: préfixes, prépositions et scalarité}

Selon Whittaker (2002:1, 21-28), la scalarité consiste à décomposer un procès, un état ou une propriété en degrés. La notion de scalarité est étroitement liée à celle de comparaison, étant donné l'implication d'un standard ou d'un étalon par rapport auquel les objets s'ordonnent, suivant le degré de possession d'une certaine propriété (Sapir 1944). Une des expressions les plus symptomatiques de la scalarité est l'intensification. Ainsi, très fort, par exemple, sera ordonné plus haut que fort et assez fort dans une échelle de force ${ }^{1}$. Contrairement à ce que l'on peut croire, l'intensification implique une gradation d'une propriété à la fois vers le haut (amplification) ou vers le bas ('downtoning') (Bolinger 1972:17).

Adler et Asnes (2004, 2007a, 2007b, à paraître) ont traité de la modification scalaire dénotée par des prépositions telles que au-delà de et jusqu'à. Cette contribution étend le champ de notre recherche afin d'explorer la part des préfixes dans la gradation. Plus précisément, nous nous intéressons ici à la possibilité pour le morphème libre au-delà de (qualifié au-delà de ce qu'on pouvait attendre) et le morphème lié sur- (surqualifié) de fonctionner comme des opérateurs de gradation.

Il existe bien sûr d'autres prépositions et préfixes capables d'opérer une gradation ${ }^{2}$ :

- $\quad$ Prépositions: jusqu'à, au-delà de, au-dessus de, au-dessous de

- $\quad$ Préfixes: sur-, sous-, mini-, maxi-, super-, hyper-, hypo-, ultra-, extra-, archi-, méga.

Cette étude porte exclusivement sur les analogies et les différences de la scalarité telle que dénotée par la préposition au-delà de et le préfixe sur- (cf. (1)-(2)):

Doué au-delà de toute mesure

\section{Surdoué}

Comment motiver le choix de ces items particuliers? Plus précisément, pourquoi ne pas comparer les divers usages de sur- en tant que préposition et préfixe? Par ailleurs, pourquoi ne pas opposer la préposition au-delà de au préfixe trans- qui, à première vue, parait plus apte à la comparaison que sur-?

Si l'on tient compte de la portée de cette étude, qui traite des opérateurs de scalarité, on se contentera d'une seule réponse: la préposition sur comme le préfixe trans- dénotent, tous les deux, des valeurs spatiales et sont donc immédiatement exclus. Seul le préfixe sur-, mais non la préposition, fonctionne comme opérateur de gradation. Pour sa part, le préfixe trans- (comme dans: transatlantique ou transsibérien) ne dénote que le sens spatio-temporel.

Nous procéderons en quatre temps: dans la première section il sera traité des différences sémantiques relevant de ces deux types de modification - par préposition et par préfixe. Les types d'échelle selon lesquelles la modification est opérée seront passés en revue dans la section 2. La troisième partie examine les restrictions imposées par au-delà de et sur-sur le type de prédicat. La dernière section oppose au-delà de à sur- dans le but d'explorer leurs valeurs discursives, car il semble que leur synonymie n'est pas garantie. 


\section{Sémantique de au-delà de et de sur-}

\subsection{Sémantique de la préposition au-delà de}

Selon le Trésor de la Langue Française, au-delà de connaît trois usages principaux: spatial, temporel et scalaire. Le dénominateur commun de ces trois valeurs serait la notion d'éloignement par rapport à un point de référence posé ou, en d'autres termes, un dépassement d'une limite. Cette limite varie selon la valeur du point de référence exprimé par le GN dans la portée de au-delà de:

\section{Dépassement d'un site spatial pris comme point de référence (océan)}

$$
\text { Habiter au-delà de l'océan }
$$

\section{Dépassement d'un intervalle temporel ( 8 heures par jour)}

$$
\text { Travailler au-delà de } 8 \text { heures par jour }
$$

En ce qui concerne les GPréps dont la tête est l'opérateur scalaire au-delà de, il y a un dépassement de la valeur standard et contextuellement définie d'un procès, d'un état ou d'une propriété (cf. Adler et Asnes 2007a, 2007b) :

(5) Paul est heureux au-delà de ce qu'on pouvait attendre

(6) Aimer au-delà de toute expression

(7) Courageux au-delà de toute mesure

En (5)-(7), au-delà de fonctionne comme un opérateur établissant une relation entre une propriété, un état ou un événement et un degré sur une échelle, tel que le degré en question (donné par au-delà de XP) est supérieur au degré de référence de cette propriété, cet état ou ce procès.

Par exemple, la proposition en (5) est vraie si, et seulement si, il y a une propriété d'être heureux attribuée à Paul dont le degré de référence est représenté par de ce qu'on pouvait attendre, et si la position de Paul sur l'échelle de bonheur est supérieure à celle qu'on pouvait attendre.

\subsection{Sémantique du préfixe sur-}

Le préfixe sur- fonctionne comme opérateur scalaire lorsqu'il est joint à des verbes, des adjectifs, des participes et des noms déverbaux et déadjectivaux.
a. surprotéger
b. surcomplet
c. surprotégé
d. surprotection

Cette section compare les usages prépositionnels et préfixationnels de sur(-). Le préfixe polyfonctionnel sur- a évolué à partir d'une préposition essentiellement spatiale. Comme notre étude traite exclusivement de la valeur scalaire de ce préfixe, les valeurs de la préposition correspondante ne seront fournies qu'à titre de comparaison.

\subsubsection{La préposition sur}

Selon le $T L F$, la préposition sur est extrêmement polysémique. Nous ne mentionnons ici que ses valeurs les plus prototypiques ${ }^{3}$ :

\section{Spatiale}


a. Antonyme de sous: sur la table

b. Point d'arrivée (cible): marcher sur Rome, se précipiter sur quelqu'un

2. Temporelle ou temporelle-causale : sur le matin, sur demande

3. Sens abstraits - point de référence (mesure, quantité): sur dix mètres, avoir avantage sur quelqu'un

\subsubsection{Le préfixe sur-}

Selon le même dictionnaire (TLF), le préfixe sur- connaît les sens suivants:

\section{Sens spatiaux}

a. Supériorité

(9) surtitre, surmaillot, survoler; surplomber, surrénal

b. Hiérarchie ${ }^{4}$

(10) surarbitre, surexpert, surclasser, surpasser, surhumain, surnaturel

\section{Temporel - Postériorité}

(11) survivre, surlendemain

\section{Itération, répétition}

(12) surimposer, surimprimer, sursemer, surdorer, surinfection, surtaxation (taxation supplémentaire)

\section{Valeur scalaire}

a. Excès

(13) surabondance, surcapacité, surpuissance, surpesanteur, survitesse, suralimenter, surchauffer, surexploiter, surestimer, surévaluer, surpayer, surtaxer, surdoué, surexcité, surpeuplé, surdéveloppé, surendetté, surnourri, surproduction, suradministration, surconsommation, suremploi, surirritation, surprotection

\section{b. Usage hyperbolique (exagération) dans le langage familier}

\section{(14) Le bus était surcomplet ${ }^{5}$}

On voit donc que contrairement à la préposition sur, le préfixe sur-fonctionne comme intensifieur. Selon Quirk et alii (1972), les intensifieurs se repartissent en trois sous-classes sémantiques: emphasizers, amplifiers, downtoners. Selon cette typologie, sur- appartient à la catégorie des amplifieurs qui opèrent une gradation vers le haut à partir d'une norme préétablie. Mais le préfixe attaché à un prédicat gradable fait plus qu'une simple intensification: il dénote, selon Bolinger (1972) et Quirk et alii (1972), l'excès ou l'idée de 'trop'. Ceci le rend apte à un changement de la valeur discursive-connotative du prédicat. Contrairement à d'autres préfixes intensifieurs, sur- paraît être le seul à pouvoir ajouter une connotation négative à l'item modifié. Pour ne donner qu'un exemple rapide, tandis que protéger semble être un mode de comportement positif ou du moins non-marqué, surprotégér n'est pas recommandé par les psychologues.

Mentionnons en passant que ceci s'observe en anglais également: le prédicat préfixé overoptimistic traduit une négativité qui est complètement absente dans le cas du radical non préfixé optimistic. Il en va de même pour les paires sweet-oversweet, generous-overgenerous ${ }^{6}$.

Les choses, on le verra bientôt (section 4), sont malheureusement plus complexes que cela: d'une part, dans les usages hyperboliques, sur- peut ne pas modifier la valeur connotative du prédicat et sert seulement d'intensifieur (en ce sens qu'il va dans le même sens que le prédicat (cf. ex. (14)). D'autre part, 
dans les usages identifiés comme appartenant à l'excès, il est possible de trouver des cas où la valeur fondamentale du radical n'est pas modifiée. Comparons deux prédicats désignant l'excès, tels que surdoué et surqualifié: contrairement à surqualifié, qui signifie "excessivement qualifié" et peut être utilisé négativement, surdoué signifie "extrêmement doué" et préserve la valeur positive du radical. Pourquoi? Afin de répondre à cette question, nous devons considérer les propriétés sémantiques du radical. Tandis que doué est un prédicat de propriété permanente (individual-level predicate), qualifié est un prédicat de propriété transitoire (stage-level predicate). Ces propriétés sont préservées lors de l'addition du préfixe sur-. Qui plus est, surdoué est un adjectif intransitif qui n'a besoin d'aucun point de référence (donc autonome). D'autre part, qualifié et surqualifié sont transitifs et ne sont pas autonomes, en ce sens qu'on est qualifié toujours pour quelque chose. Par conséquent, on peut être surqualifié pour un poste mais sousqualifié pour un autre.

De plus, nous avons suggéré que sur-peut alterner dans les usages hyperboliques avec super- ou hyper-, mais ceci ne suggère pas automatiquement qu'ils seront des synonymes parfaits :

\section{a. Superqualifié, hyperqualifié \\ b. Surqualifié}

Alors que superqualifié et hyperqualifié ont une connotation positive, et représentent une forme familière d'exagération, surqualifié peut être utilisé négativement. Dans le cas de sur-, il y a un standard qui est institutionnellement imposé et le surpassement de ce standard risque de créer une situation désavantageuse pour ladite institution. Au contraire, superqualifié et hyperqualifié expriment des jugements subjectifs qui n'impliquent aucune norme institutionnalisée.

Quant à hyperdoué/ superdoué vs. surdoué, les trois adjectifs dénotent, au contraire, la même valeur et il n'y a pas de renversement de la valeur connotative. Ces trois adjectifs sont utilisés pour décrire une personne dont les capacités intellectuelles sont supérieures à la moyenne, et donc intensifient le degré du prédicat en impliquant un standard de comparaison.

Et pour esquisser un tableau encore plus fidèle, il faudra encore prendre en compte les cas où ces soidisant synonymes sont en distribution complémentaire: par exemple hypercorrection est un terme utilisé en grammaire tandis que surcorrection appartient au domaine de l'ophtalmologie ${ }^{7}$.

\section{Types d'échelle}

Une différence majeure entre la modification scalaire opérée par les morphèmes libres (prépositions) et celle opérée par les morphèmes liés (préfixes) relève du type de l'échelle selon laquelle le prédicat est modifié. Plus précisément, dans le cas de la préposition, le prédicat modifié est évalué selon une échelle explicitement posée. Cette échelle peut être contrainte ou non par les propriétés lexicales du prédicat (cf. Adler et Asnes 2007a, 2007b). Les échelles quantitative et qualitative (cf. exemples (16), (17)), sont ainsi contraintes par le type de prédicat, à savoir un prédicat quantitatif est mesuré selon une échelle quantitative, tandis qu'un prédicat qualitatif sera mesuré selon une échelle d'intensité.

\section{Echelle quantitative $(50 \mathrm{~kg})$ :}

(16) Maigrir au-delà de $50 \mathrm{~kg}$

Echelle qualitative (amour - adoration: relation hyperonyme /hyponyme):

$$
\text { Aimer au-delà de l'adoration }
$$

Au contraire, l'échelle épistémique est compatible avec tous les types de prédication

\section{Echelle épistémique (externe) (toute expression):}

$$
\text { Aimer/ maigrir au-delà de toute expression }
$$


Contrairement aux prépositions, la modification scalaire préfixationnelle repose uniquement sur les propriétés lexicales du prédicat modifié et il n'y a aucune autre échelle explicitement posée (cf. (2), où la modification exploite l'échelle inhérente du prédicat doué).

Il en va de même pour stressé - surstressé, stimulé - surstimulé, excité - surexcité où sur- intensifie la propriété lexicale d'être stressé, stimulé ou excité et dénote que le degré 'acceptable' du stress, de la stimulation et de l'excitation a été dépassé (à l'extrémité). Autrement dit, nous avons ici un jugement de valeur: le niveau du stress etc. est mesuré par rapport à une norme d'acceptabilité. Précisément à cause de l'absence d'une échelle explicitement posée, les usages préfixationnels sont moins productifs en comparaison avec les usages prépositionnels. Ainsi, il est impossible de trouver un équivalent préfixationnel pour chaque usage prépositionnel:

$$
\text { Aimer au- delà de l'adoration - *suraimer }
$$

\section{Types de prédicats}

\subsection{Prédicats compatibles avec au-delà de}

En ce qui concerne les prédicats modifiés par les syntagmes introduits par au-delà de, il n'y a pas de restriction sur la catégorie syntaxique: le terme modifié peut être un adjectif, un nom ou un verbe à condition que ceux-ci soient scalaires. Sémantiquement parlant, les prédicats modifiés peuvent appartenir aux catégories suivantes ${ }^{8}$ :

- Prédicats à échelle ouverte/ fermée ${ }^{9}$

Les prédicats à échelle fermée sont compatibles avec des modifieurs proportionnels tels que complètement, partiellement, à moitié. Ainsi, remplir est un prédicat à échelle fermée dont le degré maximal est lexicalement inhérent. Par contre, dans le cas de l'échelle ouverte (aimer, heureux), aucun degré maximal n'est associé au prédicat (*complètement, partiellement, à moitié).

\section{Echelle ouverte}

(20) Heureux au-delà de toute expression

\section{Echelle fermée}

(21) Remplir la salle au-delà de sa capacité maximale

- Prédicats de propriété permanente (individual-level) et transitoire (stage-level) (cf. Kratzer, 1995)

\section{Prédicats de propriété permanente}

(22) Beau au-delà de toute expression

\section{Prédicats de propriété transitoire}

(23) Heureux au-delà de toute expression

- Prédicats psychologiques

(24) Aimer au-delà de toute expression

- Prédicats d'activité (Vendler, 1967)

(25) Communiquer au-delà des mots

- Prédicats d'accomplissement (Vendler, 1967)

(26) Remplir la salle au-delà de sa capacité maximale 
- Prédicats d'achèvement (Vendler, 1967)

\subsection{Prédicats compatibles avec sur-}

Les paragraphes précédents montrent que les GPréps scalaires introduits par au-delà de dénotent un haut degré et n'imposent aucune restriction sur la catégorie syntaxique du prédicat modifié, à condition que ceux-ci soient scalaires. En ce qui concerne le préfixe sur-, lorsqu'il est attaché à des prédicats non scalaires, il ne dénote pas l'intensité. Sur- dans surimprimer ajoute un sens itératif ('imprimer sur quelque chose qui est déjà imprimé'). Surinfection, quant à lui, signifie 'infection secondaire conséquente à la primaire $^{\prime 10}$.

Lorsque sur- est attaché à des prédicats scalaires, à l'exemple des prépositions, il est compatible avec des adjectifs, des noms et des verbes. Sémantiquement parlant, les prédicats modifiés par ce préfixe peuvent appartenir aux mêmes catégories que les prédicats modifiés par la préposition:

- Prédicats à échelle ouverte/ fermée

\section{Echelle ouverte:}

(28) Surcontent, surdoué

Echelle fermée:

(29) Surremplir la salle, surcharger le camion

- Prédicats de propriété permanente (individual-level) et transitoire (stage-level) (cf. Kratzer, 1995)

Prédicats de propriété permanente:

(30) Surdoué, suractif

Prédicats de propriété transitoire

(31) Surfatigue, surqualifié, surexcité, surirrité, suroccupé

- Prédicats psychologiques

(32) Surexciter, surirriter

- Prédicats d'activité (Vendler, 1967)

(33) Surfumer

- Prédicats d'accomplissement (Vendler, 1967)

(34) Surremplir, surperformer

Nous avons déjà évoqué le changement potentiel de la valeur connotationnelle du prédicat modifié par le préfixe sur-. Il est temps, à présent, de se demander si ce changement est corrélé au type du radical. En d'autres termes, le type de prédicat serait-il responsable de la connotation négative?

Si l'on compare des prédicats tels que surqualifié et surdoué, l'on pourrait croire que la dichotomie stage/individual-level est à la base de l'effet négatif. Ainsi, surqualifié qui est un prédicat de propriété provisoire (stage-level) peut être utilisé négativement, pour disqualifier quelqu'un, tandis que surdoué qui est un prédicat de propriété permanente (individual-level) transmet une valeur positive. Cependant, si on compare surdoué à un autre prédicat de propriété permanente comme, par exemple, surprotecteur, on constate que surprotecteur est susceptible de changer sa valeur connotationnelle fondamentale, contrairement à surdoué: doué est considéré comme une propriété positive, recommandable, et c'est le cas 
aussi de surdoué. Protecteur peut être considéré comme une propriété positive alors que surprotecteur devient une propriété problématique.

D'autre part, le même prédicat peut changer de connotation selon qu'il réfère à des propriétés physiques ou psychologiques. Par exemple, surperformant appliqué à une voiture, un moteur ou un PC sera vu comme une propriété positive, tandis qu'un collègue surperformant, tout en étant un trésor pour le cadre administratif, peut ne pas être bien vu des autres employés puisqu'il introduit un élément compétitif dans le mauvais sens du terme. Il en va de même pour suractif: dit d'un médicament ou d'un corps chimique il signifie "dont les effets sont très puissants ou ont été renforcés" (oxygène suractif); dit d'une personne il signifie "dont l'activité est débordante" (un homme d'affaires suractif $(T L F)$ ). Il s'avère que cette valeur additionnelle/ connotationnelle est conditionnée contextuellement, à savoir le même item peut être soit positif soit négatif dans différents contextes.

En fait, la connotation négative ou positive des termes préfixés par sur- relatifs à des personnes dépend de l'attitude respective du locuteur par rapport à la personne évaluée: tout dépassement de la norme peut déclencher chez les autres soit une attitude favorisante soit une attitude dépréciative, selon que cela coïncide ou non avec les intérêts de l'énonciateur. Cette attitude ambivalente par rapport à des propriétés jugées comme superlatives ne singularise pas uniquement l'emploi des termes préfixés par sur- et relève des considérations extra-linguistiques, liées à la psychologie humaine. Prenons, par exemple, la propriété d'être perfectionniste, dont le spectre des dénotations varie d'un perfectionnisme sain et positif jusqu'à un perfectionnisme négatif et pathologique. Ainsi, dans certains contextes "perfectionniste" veut dire "minutieux", "scrupuleux", "soigneux", "attentif" et dans d'autres "extrême", "excessif", "obsessionnelcompulsif".

Considérons encore (35) et (36). En (35) le changement possible de connotation est lié à la cause de la surexcitation: en (35a) le fait d'être surexcité affecte négativement la personne, alors qu'en (35b) le fait d'être surexcité affecte positivement la personne. En (36) surqualifié exprime un dépassement d'une norme et sert donc de base au refus d'emploi (étant donné l'inadéquation du candidat à un certain poste), mais l'évaluation s'avère désavantageuse (36a) ou avantageuse (36b) pour la personne qualifiée:

Surexcité:

a. Votre fils n'a pas réussi à s'endormir. Il était surexcité.

b. Votre fils est surexcité à l'idée de partir à la Nouvelle Orléans.

\section{Surqualifié:}

a. Vous ne pouvez pas être embauché pour ce poste parce que vous êtes surqualifié

b. Ne gaspillez pas votre temps, vous êtes surqualifié pour ce poste. En revanche, j'ai un autre poste qui pourrait vous intéresser.

Du point de vue de la force argumentative, en l'absence de contexte, le candidat ayant reçu comme réponse "vous êtes surqualifié pour ce poste", ne peut pas, en principe, être tout à fait sûr des intentions de l'intervieweur: celui-ci est-il en train de justifier le refus ou plutôt de proposer un emploi plus adéquat dans l'organisation? Dans l'esprit de la théorie d'Anscombre et Ducrot (1983), le changement de "connotation" peut donc être corrélé à la force ou l'orientation argumentative de l'énoncé, c'est-à-dire à l'ensemble de conclusions visées.

Nous voyons donc l'influence discursive sur le changement possible de connotation. La notion d'excès exprimée par sur- ou la transition subtile de "plus que" à "trop" est aussi discursive. Qui plus est, certaines qualifications sont institutionnellement ancrées ou standardisées et ainsi considérées comme des concepts (surprotecteur - psychologie, surqualifié - marché de travail). Dans ces cas, la valeur connotationnelle de l'item est fixe.

Il reste encore la question d'antonymie: comment se fait-il que surdévelopper, surexciter, surcomplet, surabondant existent, à l'encontre de *sous-développer, *sous-exciter, *sous-complet, *sous-abondant? Ceci semble être lié au type de prédicat: développer est télique et impose, en tant que tel, une borne 
finale. Ce serait en effet bizarre d'ajouter sous qui empêche la finalisation du procès (i.e. en contredisant les propriétés aspectuelles inhérentes du prédicat). Ceci expliquerait, également, pourquoi les participes passés de tels verbes sont tout de même possibles (sous-développé): le participe passé exprime l'état résultant et dans ce cas il est possible de juger si le procès a abouti à son terme ou non. D'autre part, sur/sous-estimer ne posent pas de problème puisqu'ils sont atéliques (activités vendleriennes) et, en tant que tels, n'ont pas de terme inhérent. A chaque instant de l'activité d'estimer, le procès est vrai ${ }^{11}$. Le préfixe sous-, dans ce cas, n'est pas contradictoire avec les propriétés aspectuelles du prédicat. En fait, dans le cas de sous-developpé, sous- signifie "pas complètement", dans sous-estimé, qui est atélique, sous- signifie "pas assez" (l'équivalent de télique dans le domaine verbal est l'échelle fermée dans le domaine adjectival - surcomplet).

\section{Modification par morphèmes libres et par morphèmes liés: la question de la connotation}

Nous sommes tentées d'utiliser l'exemple (37) ci-dessous pour montrer que quand la modification est opérée par des morphèmes libres, comme dans le cas d'une échelle épistémique, les inférences sont différentes des cas où la modification est opérée par des morphèmes liés: dans le premier cas, la valeur connotationnelle du radical est préservée et, bien sûr, intensifiée par le modifieur. Dans le deuxième cas, il peut y avoir un changement de valeur connotationnelle lié à la possibilité d'un jugement négatif ou à une incompatibilité avec un standard préalablement fixé.

\section{Qualifié au-delà de ce qu'on pouvait attendre / surqualifié}

Dans le marché du travail, si une personne est surqualifiée, il est fort possible qu'elle ne soit pas admise au poste auquel elle a postulé. En revanche, si elle est 'qualifiée au-delà de ce qu'on pouvait attendre', ceci signifie qu'elle a fait une impression extraordinaire sur ses futurs employeurs et sera très probablement embauchée. A première vue, et comme on l'a signalé dans les paragraphes précédents, il paraît que surdéclenche une inférence négative liée à la notion d'excès tandis que au-delà de marque tout simplement le haut degré. 'Excès' et 'haut degré' sont des sens évaluatifs et en tant que tels établissent une comparaison par rapport à une certaine norme sur telle ou telle échelle de référence qui est dépassée. Ceci dit, ces deux significations diffèrent par leurs effets pragmatiques: contrairement au haut degré, l'excès implique un dépassement négatif d'une limite (cf. Amiot 2004).

Malheureusement, la situation, on l'a vu, est beaucoup plus complexe. En ce qui concerne la préposition, cette valeur connotationnelle varie selon le type de l'échelle. Quand le prédicat est modifié selon une échelle épistémique (externe au prédicat), sa valeur connotationnelle dépend de la nature positive ou négative du jugement. Comparons:

$$
\begin{aligned}
& \text { a. Qualifié au-delà de ce qui est nécessaire } \\
& \text { b. Qualifié au-delà de ce qu'on pouvait attendre }
\end{aligned}
$$

Dans (38), il y a un mapping entre l'échelle de qualification et celle d'un jugement épistémique (échelle de nécessité dans (a) ou d'attente dans (b)) : une valeur sur l'échelle de qualification est mise en rapport avec une valeur sur l'échelle de nécessité ou d'attente. Nécessaire et ce qu'on pouvait attendre sont pris en l'occurrence comme le degré standard d'être qualifié. Etant donné que au-delà de ce qu'on pouvait attendre exprime un jugement positif, qualifié est évalué positivement. D'autre part, au-delà de ce qui est nécessaire peut être perçu comme un jugement négatif, et dans ce cas l'ensemble sera aussi négatif (ou au moins désavantageux pour la personne qualifiée).

Dans le cas d'une échelle qualitative, la modification par au-delà de se fait selon une échelle interne au prédicat et va dans le même sens de la valeur connotationnelle fondamentale du prédicat:
a. Aimer au-delà de l'adoration (intensification dans un sens positif)
b. Terrifier au-delà de l'horreur (intensification dans un sens négatif) 
Dans (39a), adoration et aimer appartiennent au même champ lexical et se placent donc sur la même échelle, celle de l'affection. L'intensification découle du fait d'une hiérarchisation lexicale : adoration représente un degré supérieur de ce sentiment par rapport à aimer (adorer est un hyponyme d'aimer ou, en parlant en termes de sèmes, plus spécifié que aimer). Ainsi, la modification par adoration exploite l'échelle interne du prédicat aimer. En (39b) on observe le même processus : l'intensification par horreur exploite l'échelle interne du prédicat terrifier.

Finalement, quant à l'échelle quantitative, il n'y a pas de jugement qualitatif et les quantités exprimées peuvent inférer des jugements positifs ou négatifs, en rapport avec notre savoir extra-linguistique.

En ce qui concerne les préfixes, surqualifié semble à première vue une propriété problématique, comme il a été précédemment montré, particulièrement quand il sert de base à un refus d'embauche. Cependant, il faut reconnaitre qu'il est préférable d'être surqualifié que sous-qualifié.

Il reste un dernier aspect à prendre en considération (cf. (40)- (41)):
a. Surestimer
b. Estimer au-delà de sa valeur
c. Estimer au-delà de toute expression
a. Surdoué
b. Doué au-delà de la moyenne
c. Doué au-delà de toute expression

Ces exemples montrent que la modification prépositionnelle peut aussi exploiter l'échelle interne. Le même résultat peut ainsi être obtenu par la modification préfixationnelle et prépositionnelle: (a) et (b), mais non (c), exploitent l'échelle interne (inhérente) du prédicat.

Selon Noailly (1999:37-39) les quatre préfixes d'intensité que sont super-, hyper-, archi-, extra- dénotent un sens équivalant à celui de très. Elle distingue super- et extra-, d'une part, qui intensifient des qualités positives (super-fort ou extra-fort) ${ }^{12}$. Ces préfixes sont mélioratifs à tel point qu'ils peuvent être utilisés tout seuls (Cette fille est super. Ce garçon est extra). Les préfixes hyper- et archi- peuvent, d'autre part, intensifier à la fois des propriétés positives et négatives (archidoué vs. archi-ignorant, hyperdoué vs. hyper-nul). Nous observons donc que selon Noailly, les préfixes super-et extra- sélectionnent des adjectifs positifs et l'intensification qu'ils opèrent va aussi dans le sens positif. Hyper- et archi- n'ont pas de restriction de sélection sur le type de prédicat et leur intensification va dans le sens de la prédication: si paresseux est vu comme une propriété négative, hyper-paresseux sera encore plus négatif. Pour ce qui est de sur-, Noailly remarque que contrairement à ces quatre préfixes d'intensité, sur- ne marque pas l'intensité toute pure, mais l'intensité excessive et est ainsi équivalent de trop plutôt que de très. Par conséquent, sur- n'appartient pas à la même classe que ces quatre préfixes.

Nous avançons que sur-, comme hyper- et archi-, peut sélectionner à la fois des prédicats dénotant des propriétés vues comme positives (surdoué) ou négatives (surstressé), mais, contrairement à hyper-et archi-, l'intensification opérée ne va pas toujours dans le sens de la prédication non-préfixée: ainsi qualifié est une propriété positive, mais surqualifié est une propriété qui risque de devenir problématique dans le milieu professionnel. Dans le cas des qualités originellement négatives, l'intensification ne change pas l'orientation négative de la qualité. Pour ce qui est de l'intensification des qualités originellement positives, un changement éventuel est observable. Ceci est dû à des considérations extra-linguistiques: l'excès exprimé par sur- signifie en effet (comme le fait remarquer Noailly) un dépassement d'une norme (institutionnelle, comportementale, "behavioristique", etc.), mais ce dépassement peut être valorisé par les uns et méprisé par les autres (ex. un collègue surperformant est apprécié de son chef mais parfois méprisé des autres employés). 


\section{Conclusion}

La scalarité consiste en la dénotation d'une propriété qui peut être possédée selon un certain degré (Rivara, 1990). La présente étude a traité de la modification du degré naturel de prédicats scalaires par des morphèmes libres et liés. La question de la valeur négative a surgi lors de la comparaison de la modification scalaire par préfixes et par prépositions. Selon Sapir (1944) l'aspect kinesthésique de la scalarité implique le fait d'ordonner des éléments du plus petit au plus grand: le mouvement ascendant est généralement associé à un effet favorable (amplification), tandis que le mouvement descendant est associé à un effet défavorable (réduction). La présente étude expose plusieurs cas de mouvement orienté vers la limite supérieure dont l'inférence est pourtant non-favorable, ce qui suggère que la valeur connotationnelle peut ne pas dépendre de l'orientation de l'échelle (ascendante ou descendante), mais est, par contre, contrainte par des éléments contextuels/discursifs et les types de prédicat et de l'échelle.

\section{Dictionnaires}

Merriam Webster

Trésor de la langue française informatisé - TLF

\section{Références bibliographiques}

Adler, S. \& Asnes M. (2004). Les compléments de degré en jusqu'à. Travaux de Linguistique, 49, 131-157.

Adler, S. \& Asnes M. (2007a). Le monde d'au-delà : une dimension scalaire. Travaux de linguistique, 54.1, $29-42$.

Adler, S. \& Asnes M. (2007b). Au delà de l'espace et du temps : étude comparée d'un marqueur de gradation. Lingvisticae Investigationes, 30.2, 163-179.

Adler, S. \& Asnes M. (2010, in press). Prépositions au service de la scalarité. In Hadermann, P., Inkova, O. (eds), Approches de la scalarité, Recherches et Rencontres, 28, Genève: Droz, 223-247.

Amiot, D. (2004). Haut degré et préfixation. In Lefeuvre, F., Noailly, M. (éds) Intensité, Comparaison, Degré 1. Travaux Linguistique du Cerlico, 17, 91- 104.

Anscombre, J.C. \& Ducrot, O. (1983). L'argumentation dans la langue. Liège-Bruxelles : Mardaga.

Bolinger, D. (1972). Degree Words. The Hague : Mouton de Gruyter.

Ducrot, O. (1996). Lexique et gradualité. In Alonso, E., Bruna, M. et Munos, M. (éds), La lingüistica francesa: gramatica, historia, epistemologia. Séville. 191-205.

Kennedy, Ch. (2001). Polar opposition and the ontology of 'degrees'. Linguistics and Philosophy, 24, 33-70.

Kennedy, Ch. \& McNally, L. (1999). From Event Scales to Adjective Scales: Degree Modification in Deverbal Adjectives. In Proceedings of SALT 9. Ithaca : Cornell Linguistic Club Publication.

Kenny, A. (1963). Action, Emotion and Will. London : Routledge and Kegan.

Kratzer, A. (1995). Stage-level and individual-level predicates. In Carlson G.N. et Pelletier, F.J.(eds), The Generic Book. Chicago : The University of Chicago Press, 125-175

Noailly, M. (1999). L'adjectif en français. Paris : Ophrys, Collection L'essentiel.

Mandelbaum, D. G. (éd.) (1949 [1958]). Selected writings of Edward Sapir in Language, Culture and Personality. Berkley and Los Angeles : University of California Press.

Piñón, Ch. (2000). Happening gradually. In Proceedings of the Berkeley Linguistics Society 26.

Quirk R., Greenbaum S., Leech G., Svartvik J. (1972). A Grammar of Contemporary English. London : Longman.

Rivara, R. (1990). Le système de la comparaison. Paris : Editions de Minuit.

Vendler, Z. (1967). Linguistics in philosophy. Ithaca, New York : Cornell U.P. 
Whittaker, S. (2002). La notion de gradation, application aux adjectifs. Publications Universitaires Européennes, Peter Lang.

1 La scalarité peut être quantitative si elle réfère à une échelle de nombres, ou qualitative si elle réfère à une échelle de propriétés. Rivara (1990) considère la scalarité qualitative comme une 'quantification évaluative' impliquant une échelle subjective.

${ }^{2}$ La présente étude est conduite dans le cadre théorique de la sémantique de scalarité qui a profité d'une recherche abondante pendant ces dernières années (Ducrot 1996, Kennedy et McNally 1999, Piñón 2000, Kennedy 2001, Whittaker 2002).

${ }^{3}$ Exemples issus du $T L F$.

${ }^{4}$ La notion de spatialité liée à la sous-catégorie de 'Hiérarchie' doit être prise au sens figuré.

${ }^{5}$ Sur-peut alterner avec super- ou hyper-.

${ }^{6}$ En tant que préposition, over n'est pas l'équivalent de sur, mais en tant que préfixes, les deux traduisent la même idée d'intensité. Cela dit, sur- est beaucoup plus restreint que son homologue anglais over-, lequel est employé abondamment pour les intensifications quantitative et qualitative:

a. Overeat - manger plus que nécessaire (quantité)

b. Overgenerous - généreux de façon intense (qualité)

En français moderne, hyper- et super- sont beaucoup plus productifs que sur-et semblent être plus aptes que ce dernier à la comparaison avec over- du point de vue de la spontanéité et de la productivité. Ainsi, tandis que overhappy est parfaitement acceptable en anglais, surheureux est inapplicable en français. Par contre, superheureux et hyperheureux ne sont pas exclus.

${ }^{7}$ Cette étude ne traite pas de hyper- ou super-. Ceci dit, il est intéressant d'observer le résultat obtenu par la combinaison de hyper- avec un radical non-scalaire, comme dans hypercorrection: étant donné qu'en principe il est impossible d'intensifier un prédicat non-scalaire, hypercorrection signifie en fait 'incorrection', c'est-à-dire une négation du prédicat.

${ }^{8}$ La typologie proposée pour les exemples (20) - (27) et (28) - (34) repose sur une série de critères: classe lexicale (e.g. prédicats psychologiques), classe aspectuelle (e.g. prédicats d'accomplissement ou d'activité), propriété permanente/transitoire, échelle ouverte/fermée.

${ }^{9}$ La dichotomie 'échelle ouverte/ fermée' existe parallèlement à d'autres classements: on pourrait établir une analogie entre les notions d"échelle ouverte/ fermée', appartenant au domaine de la scalarité, et celle de 'télicité/ atélicité', respectivement, appartenant au domaine de l'aspect. Ainsi, remplir est un prédicat télique à échelle fermée.

10 D'autres cas à prendre en considération:

-Surmonter: sens non compositionnel du préfixe et du radical (par conséquent, hors de la portée de cette étude).

-Incompatibilités dues au type du radical: Danser - ?surdanser; *Sur/sous-tuer (prédicats atomiques).

11 Cf. Kenny (1963).

${ }^{12}$ Toutefois, on rencontre des occurrences telles que superdébile ou supercon: l'existence de telles formations ne doit pas forcément fragiliser la thèse de Noailly étant donné qu'elles peuvent être prises dans un sens ironique, à savoir la personne qualifiée représente le meilleur exemplaire de la qualité en question. 